\title{
A FORMAÇÃO DE PROFESSORES NAS LICENCIATURAS EM EDUCAÇÃO DO CAMPO: O QUE APONTAM OS ARTIGOS A PARTIR DO PORTAL DE PERIÓDICOS CAPES
}

\author{
Edir Neves Barboza ${ }^{1}$, Renata Portela Rinaldi ${ }^{1}$, Felipe Varussa de Oliveira Lima ${ }^{2}$ \\ ${ }^{1}$ Universidade Estadual Paulista - UNESP, Presidente Prudente, SP. ${ }^{2}$ Universidade Federal da Grande Dourados, MS. E- \\ mail: edirnb@gmail.com \\ Agência de fomento: CAPES
}

\section{RESUMO}

Este trabalho integra a pesquisa de doutorado intitulada "A formação de professores e a organização do trabalho pedagógico no curso de Licenciatura em Educação do Campo (LEDUC): estudo de caso da Universidade Federal da Grande Dourados (UFGD)", vinculada ao Programa de Pós-Graduação em Educação da Universidade Estadual Paulista (UNESP), Presidente Prudente. 0 objetivo do artigo é selecionar e identificar os resultados de produções científicas localizadas para o período de 2001-2018 no Portal de Periódicos Capes/MEC, utilizando os descritores: Licenciatura, Formação Inicial, Graduação e Educação do Campo. Resultante de pesquisa bibliográfica elencamos quantitativamente as produções neste período, gerando um estado do conhecimento sobre a formação de professores nos cursos de Licenciatura em Educação do Campo, permitindo posteriormente analisar e compreender os processos formativos e suas relações com o contexto em que são realizados, as tensões e os dilemas enfrentados pelos cursos e os significados atribuídos à eles pelos sujeitos envolvidos.

Palavras-chave: Formação inicial de Professores, Licenciatura em Educação do Campo, Educação Básica, Educação do Campo.

\section{THE TEACHER'S TRAINING IN RURAL EDUCATION DEGREE COURSES: WHAT DOES THE ARTICLES POINT FROM THE CAPES PERIODICALS}

\begin{abstract}
This work integrates the PhD research entitled "Teacher training and the organization of pedagogical work in the Rural Education degree courses (LEDUC): case study of the Universidade Federal da Grande Dourados (UFGD)", linked to the Graduate Program in Education, UNESP, Presidente Prudente. The objective of this article is to select and identify the articles located for the period 2001-2018 in the Capes/MEC Periodicals Portal, using the descriptors: Teaching degree, Initial teacher training, Undergraduate course and Rural Education. Resulting from bibliographical research we quantitatively list the productions in this period, generating a state of knowledge about teacher training in the Rural Education degree courses, allowing later to analyze and understand the formative processes and their relations with the context in which they are realized, the tensions and dilemmas faced by the courses and the meanings attributed to them by the subjects involved.
\end{abstract}

Keywords: Initial Teacher Training. Rural education degree courses. Basic education. Rural education. 


\section{INTRODUÇÃO}

Este trabalho aborda parte da pesquisa bibliográfica realizada no banco de dados dos Periódicos Capes no período de 2001 a 2018 acerca do tema Licenciatura em Educação do Campo. A análise apresentada se configura, portanto, como um recorte da pesquisa "A formação de professores e a organização do trabalho pedagógico no curso de licenciatura em educação do campo: estudo de caso da UFGD", vinculada ao Programa de Pós-Graduação em Educação da Universidade Estadual Paulista (Unesp), Faculdade de Ciências e Tecnologia, Presidente Prudente.

A Educação do Campo no Brasil ganha maior visibilidade a partir dos anos de 1990, oriunda das lutas dos movimentos sociais, especialmente daqueles que lutam pelo acesso à terra, como o Movimento dos Trabalhadores Rurais Sem Terra (MST), a Comissão Pastoral da Terra (CPT), ligada à Igreja Católica, o Movimento de Mulheres do Campo (MMC) e outros que, além de buscar acesso à terra, buscaram também construir um projeto de educação voltado especificamente para atender a realidade da vida campesina. E, dentro desse contexto surge a necessidade de formar professores para atuarem nesses espaços escolares, assim, essa formação necessariamente busca alimentar o sonho de um campo vivo, produtivo, feliz e capaz de produzir conhecimento partindo de uma realidade concreta, que é a escola do campo.

Nos anos 2000, o Ministério da Educação (MEC) através da então Secretaria de Educação Continuada Alfabetização e Diversidade (SECAD) criou o Programa de Apoio à Formação Superior em Licenciatura em Educação do Campo (PROCAMPO) ${ }^{1}$. A partir desse programa verifica-se novas experiências nas universidades públicas brasileiras com a oferta desse curso. Podemos afirmar com isso, que em decorrência da criação deste programa, surge uma nova temática de pesquisa, a ser investigada nos programas de pós-graduação.

A partir desse contexto, este artigo tem como principal objetivo demonstrar a produção de artigos acerca da temática Licenciatura em Educação do Campo, para compreender e analisar o processo de formação de professores nesta licenciatura.

\section{METODOLOGIA}

O artigo apresentado se insere dentro de uma pesquisa maior, em desenvolvimento, que se caracteriza como Estudo de Caso. Para tanto, a pesquisa contempla quatro características que são essenciais em estudos dessa natureza na pesquisa qualitativa, são elas: particularidade, descrição, heurística e indução (MERRIAN, 1988).

Segundo André (2005, p.31):

Sintetizando ideias de vários outros autores, pode-se dizer que o estudo de caso do tipo etnográfico em educação deve ser usado quando: (1) há interesse em conhecer uma instância em particular (2) pretende-se compreender profundamente essa instância particular em sua complexidade e totalidade; e (3) busca-se retratar o dinamismo de uma situação numa forma muito próxima do seu acontecer natural.

Para alcançar os objetivos de um estudo de caso, é necessário que a investigação do objeto de estudo ocorra mediante uma vasta coleta de dados, daí a necessidade da busca de documentos, da observação e da aplicação de questionários e entrevistas. O fenômeno investigado é situado num determinado período temporal, e, por isso, as determinações sociais, culturais, econômicas e políticas permitem ampliar a análise qualitativa que a pesquisa exige.

A necessidade de conhecer a fundo o objeto estudado nos fez realizar essa busca, pois é importante conhecer o que se tem produzido acerca do tema, esses dados possibilitam esmiuçar o contexto da pesquisa sobre a formação do professor do campo, resultando num cenário sobre o tema.

\footnotetext{
${ }^{1}$ Para maiores informações ver Molina e Sá (2011), sobre as Licenciaturas em Educação do Campo - Registros e reflexões a partir das experiênciaspiloto (UFMG, UNB, UFBA e UFS).
} 
Os procedimentos iniciais adotados para esta coleta de dados foram: definir a base de dados e o período a ser investigado. Desse modo, definimos utilizar o Portal de Periódicos Capes/MEC, pois ele abarca um vasto número de bases de dados e periódicos importantes para a área educacional. O período de 2001 a 2018 foi definido considerando a data dos Marcos Normativos da Educação do Campo, sendo o primeiro documento o Parecer no 36, datado de 04 de dezembro de 2001 (BRASIL, 2012), chegando até o tempo presente.

Para as buscas no Portal de Periódicos Capes/MEC foram utilizados três descritores (licenciatura, formação inicial e graduação) relacionados separadamente pelo operador booleano (AND) com o descritor (educação do campo), sendo os descritores compostos por mais de uma palavra, colocados entre aspas. Assim, as seguintes estratégias de busca foram utilizadas: licenciatura AND "educação do campo"; "formação inicial" AND "educação do campo" e graduação AND "educação do campo". Desta forma realizamos três buscas, obtendo inicialmente o que chamamos de resultados (encontrados) para cada estratégia de busca. Com a intenção de refinar os resultados da busca para a temática foram utilizados os seguintes filtros: artigos, tópicos relacionados à temática de interesse, e período de publicação de 2001 até 2018, obtendo para cada estratégia de busca o que chamamos de resultados (refinados). Após o passo anterior, analisando o resumo ou parte dos artigos, obtivemos os resultados (selecionados), posteriormente comparamos estes para as três diferentes estratégias de busca e obtivemos os resultados ou artigos (inéditos), ou seja, aqueles que ocorrem em somente uma estratégia, dos quais serão analisados o seu conteúdo no aprofundamento desse estudo.

\section{RESULTADOS}

Na Tabela 1, apresentamos o número de artigos encontrados, refinados, selecionados e inéditos no Portal de Periódicos Capes/MEC a partir das estratégias de busca utilizadas como detalhado na metodologia. O maior número de artigos encontrados foi registrado para a estratégia de busca com o descritor graduação, totalizando 127 artigos. Por outro lado, após serem refinados os resultados (artigos refinados) e analisarmos os trabalhos detalhadamente foram selecionados (artigos selecionados) apenas quatro artigos com o descritor graduação. Acreditamos que número elevado de artigos encontrados para o descritor graduação teve relação com o fato do sistema de busca localizar artigos que contenham o termo pós-graduação. Do total de 259 resultados encontrados, 30 foram considerados como resultados inéditos, ou seja, são os artigos que aparecem em apenas uma das estratégias de busca, portanto, não são repetidos.

Tabela 1. Número de artigos por estratégia de busca e totais dos resultados obtidos no Portal Periódicos Capes/MEC.

\begin{tabular}{|l|cccc|}
\hline ESTRATÉGIA DE BUSCA & ENCONTRADOS & REFINADOS & SELECIONADOS & INÉDITOS \\
\hline $\begin{array}{l}\text { licenciatura AND "educação do } \\
\text { campo" }\end{array}$ & 102 & 45 & 35 & 29 \\
$\begin{array}{l}\text { "formação inicial" AND "educação do } \\
\text { campo" }\end{array}$ & 30 & 8 & 5 & 1 \\
graduação AND "educação do campo" & 127 & 37 & 4 & 0 \\
TOTAL & 259 & 90 & 44 & 30 \\
\hline
\end{tabular}

Fonte: Dados organizados a partir de pesquisa bibliográfica no Portal Periódico Capes/MEC em 2018.

O Gráfico 1 apresenta: os dados por estratégia de busca. Os resultados encontrados em ordem decrescente por descritor foram: graduação, licenciatura e formação inicial, tendo uma diferença de $37,45 \%$ entre o maior e o menor. Os resultados refinados em ordem decrescente por descritor foram: licenciatura, graduação e formação inicial, tendo uma diferença de $41,11 \%$ entre o maior e o menor. Os resultados selecionados em ordem decrescente por descritor foram: 
licenciatura, formação inicial e graduação, tendo uma diferença de 70,45\% entre o maior e o menor. Apenas dois artigos foram repetidos, localizados nas três estratégias de busca.

Gráfico 1. Número de artigos por estratégia de busca dos resultados obtidos no Portal de Periódicos Capes/MEC.

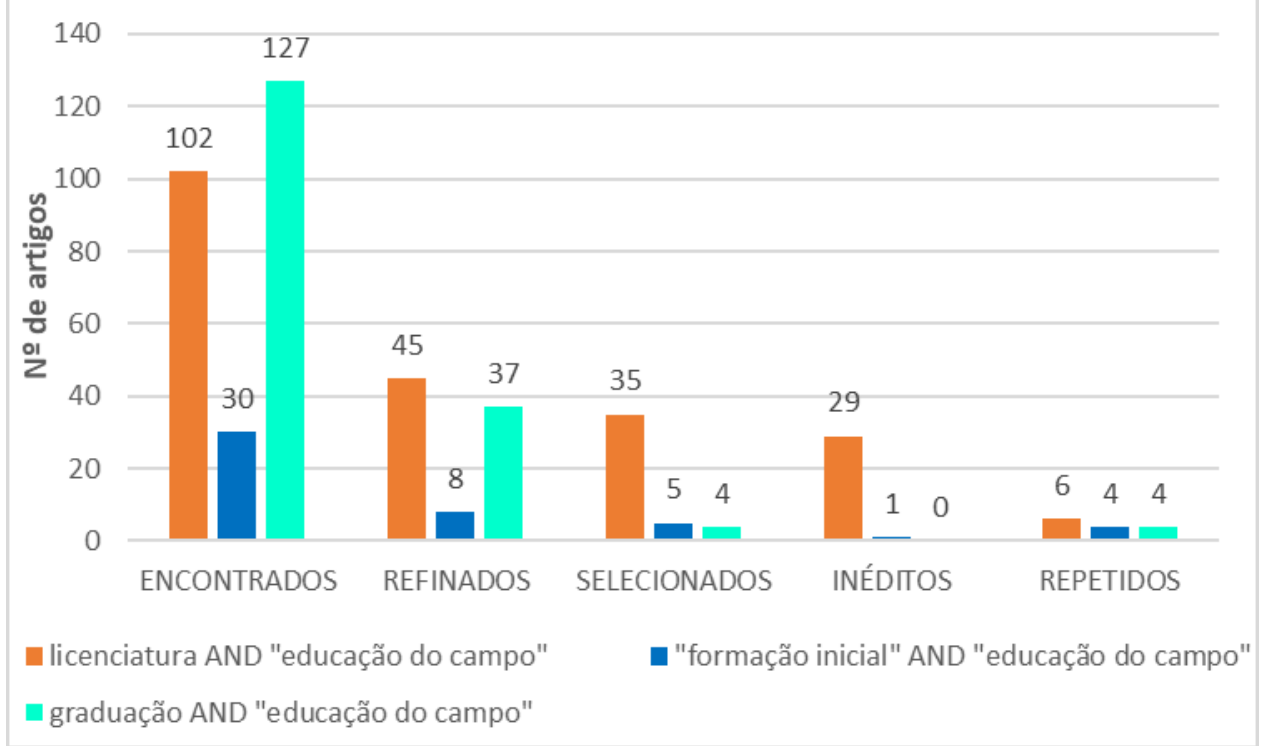

Fonte: Dados organizados a partir de pesquisa bibliográfica no Portal de Periódicos Capes/MEC em 2018.

No Gráfico 2, podemos observar o percentual dos artigos inéditos por estratégia de busca. O descritor licenciatura apresentou o maior número de produções, pois o termo está ligado diretamente as questões que circundam a formação de professores, já que os cursos são de licenciatura, não há um estranhamento nesse sentido. A estratégia com o descritor formação inicial tem um percentual pequeno, já que também aborda questões diretas da formação de professores. O termo graduação é muito abrangente, já que todos os cursos de Ensino Superior são de graduação e não apresentou nenhum artigo inédito.

Gráfico 2. Percentual de resultados de artigos inéditos por estratégia de busca obtidos no Portal Periódicos Capes/MEC.

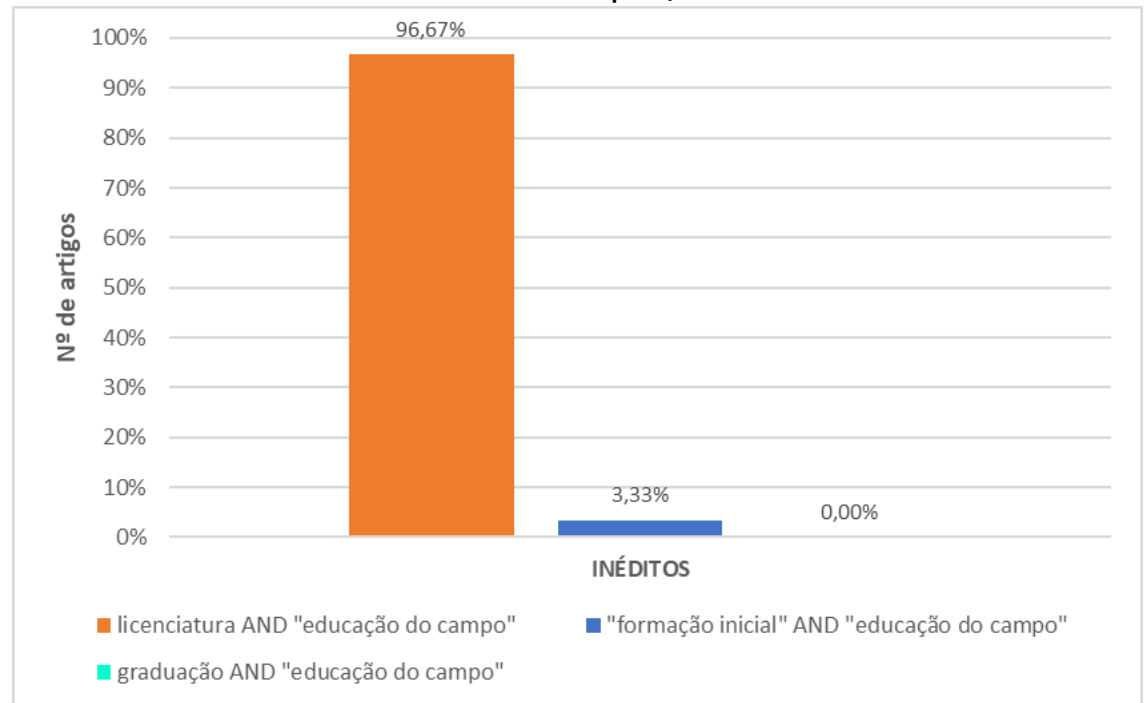

Fonte: Dados organizados a partir de pesquisa bibliográfica no Portal de Periódicos Capes/MEC em 2018.

No Gráfico 3, são apresentados os artigos inéditos, ou seja, aqueles encontrados exclusivamente em uma das três estratégias de busca utilizadas, desconsiderados os repetidos nas 
diferentes estratégias. Destes artigos inéditos, o mais antigo para o período utilizado na pesquisa, data o ano de 2008 e o mais recente, o ano de 2017. A estratégia com o descritor licenciatura teve o maior número de artigos inéditos, com tendência crescente no período 2008 até 2017. Acreditamos que este período represente o momento da finalização de teses e dissertações e, portanto, a publicação de artigos decorrentes destas.

Gráfico 3. Número de artigos inéditos para cada estratégia de busca por ano obtidos no Portal de Periódicos Capes/MEC.

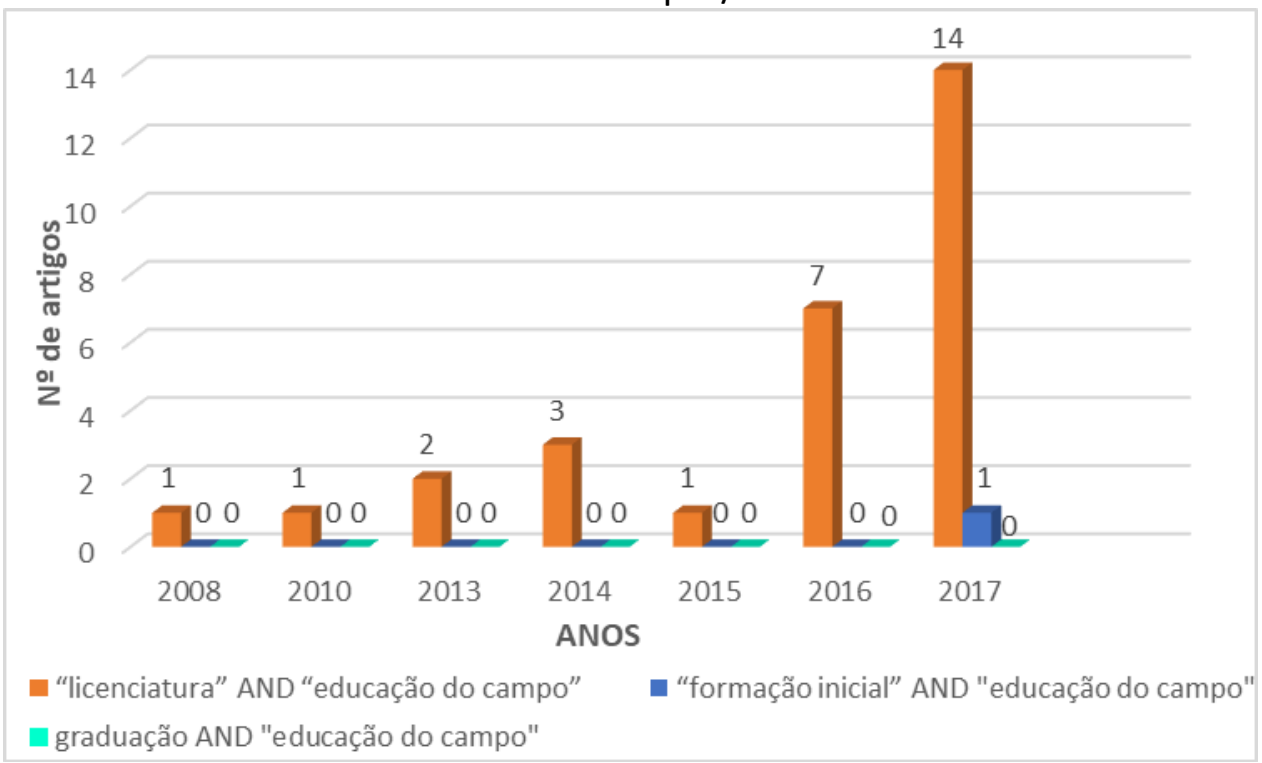

Fonte: Dados organizados a partir de pesquisa bibliográfica no Portal de Periódico Capes/MEC em 2018.

\section{DISCUSSÃO}

A análise realizada neste artigo, a partir do objetivo proposto, evidencia o cenário da produção acadêmica através de artigos selecionados no Portal de Periódicos Capes/MEC sobre a temática formação de professores com o recorte para a Licenciatura em Educação do Campo. Os resultados apontam algumas análises, que por se tratar da formação de professores refletem diretamente na realidade da educação brasileira.

Dessa maneira, voltando para a concretude dos cursos de Licenciatura em Educação do Campo, a pesquisa bibliográfica evidencia que o período de produções coincide com a implementação em 2008 dos cursos de Licenciatura em Educação do Campo nas Universidades públicas. A estratégia de busca com o descritor licenciatura resultou no maior número de artigos selecionados e artigos inéditos. Por essa razão, consideramos que este foi o melhor descritor para evidenciar as publicações sobre a temática investigada no Portal de Periódicos Capes/MEC.

O descritor formação inicial tem como significado a primeira formação. Nas buscas realizadas encontramos apenas um artigo inédito, exclusivo para esse descritor. Acreditamos que este resultado reforça a ideia do termo licenciatura ser a nomenclatura mais habitual, ao se tratar do tema formação de professores, visto pelo maior número de artigos encontrados com o descritor licenciatura.

De forma geral, o número de artigos selecionados indica que há muito ainda a ser investigado no que se refere a Licenciatura em Educação do Campo, pois se trata de pesquisa do tempo presente, considerando que esses cursos completam dez anos de implementação, nesse sentido as primeiras produções começam agora a se materializar.

O cenário representado por essa pesquisa indica a necessidade de investigação do complexo processo de formação dos professores, especificamente dos sujeitos oriundos da população camponesa. Pois, em linhas gerais, esse processo formativo deve contribuir para a vida e valores das pessoas envolvidas diretamente com a realidade das escolas do campo. 
Portanto, torna-se necessário ampliar a investigação ora apresentada, para suscitar novos elementos desse processo de formação, perpassando desde conhecer, investigar e analisar as políticas de formação de professores das escolas do campo, bem como, o contexto das salas de aula, da prática desse professor e da profissionalidade desses sujeitos.

Desse modo, podendo colaborar para a elaboração e implementação de políticas educacionais que garantam, a ampliação e a qualidade no processo de formação dos professores e das escolas do campo.

\section{CONCLUSÃO}

A temática formação de professores no Brasil não é recente, desde os anos 1950, as pesquisas têm abordado questões inerentes a esse processo. No entanto, verificamos que a partir do ano 2000, uma nova modalidade de formação de professores tem sido implementada nas universidades brasileiras, trata-se dos cursos de Licenciatura do Campo e Licenciatura Indígena. As especificidades destes cursos iniciam em suas nomenclaturas, dando a entender que trabalham preferencialmente com grupos sociais distintos, camponeses e indígenas.

Em relação ao objeto dessa investigação, a Licenciatura do Campo, tem como uma das suas especificidades, a metodologia da Alternância, desse modo, organiza as suas atividades pedagógicas, teóricas-metodológicas, nos tempos e espaço de estudo, que se dividem em: Tempo Universidade e o Tempo Comunidade.

Desse modo, esse novo processo formativo de professores no Brasil, ainda é pouco conhecido em parte do meio acadêmico e nos programas de pós-graduação. Sendo, portanto, um novo campo de investigação que pelo tamanho de sua complexidade muito ainda tem para ser explorado. As produções ainda incipientes tendem a crescer, pois também se verifica uma consolidação dessa política de formação de professores.

Essa pesquisa bibliográfica revela a necessidade e a importância de se ampliar a investigação em torno da temática Licenciatura em Educação do Campo nas produções acadêmicas nas universidades brasileiras.

O estudo também nos dá indicações do quanto se fazem necessários investimentos na área educacional e na formação de professores. Tais investimentos, pensando nos diversos programas de pós-graduação, poderiam contribuir para a realização de pesquisas que visam compreender e analisar as condições dos espaços escolares e da política de formação de professores no Brasil. Resultando assim, na valorização do magistério, na melhor formação de professores e, portanto, numa educação com qualidade social.

\section{REFERÊNCIAS}

ANDRÉ, M. E. D. A. de. Estudo de caso em pesquisa e avaliação educacional. Brasília: LiberLivro, 2005. Série Pesquisa; v. 13, 68p.

BRASIL. Ministério da Educação. Secretaria de Educação Continuada, Alfabetização, Diversidade e Inclusão - SECADI. Educação do Campo: marcos normativos/Secretaria de Educação Continuada, Alfabetização, Diversidade e Inclusão - Brasília: SECADI, 96p., 2012.

MERRIAN, S. Case study research in education: A qualitative approach. San Francisco, CA: JosseyBass.1988.

MOLINA, M. C.; SÁ L. M. (Orgs). Licenciaturas em Educação do Campo: Registros e Reflexões a partir das Experiências Piloto. Belo Horizonte: Autêntica Editora, 2011 (Coleção Caminhos da Educação do Campo; 5). 